\title{
THE FORMATION OF A POWER MULTI-PULSE EXTREME ULTRAVIOLET RADIATION IN THE PULSE PLASMA DIODE OF LOW PRESSURE
}

\author{
Ievgenita V. Borgun ${ }^{a, *}$, Mykola O. Azarenkov $^{a}$, Ahmed Hassanein $^{b}$, \\ Oleksandr F. TSEluyko $^{a}$, VAsyl I. MASlov ${ }^{a}$, DMitro L. RyABChiKovK ${ }^{a}$ \\ ${ }^{a}$ Karazin Kharkiv National University, pl. Svobody 4, Kharkov 61022, Ukraine \\ ${ }^{b}$ School of Nuclear Engineering, Purdue University, 400 Central Drive, West Lafayette, IN 47907-2017, USA \\ * corresponding author: ievgeniia.borgun@mail.ru
}

\begin{abstract}
In this paper results are presented on experimental studies of the temporal characteristics of spike extreme ultraviolet (EUV) radiation in the spectral range of $12.2 \div 15.8 \mathrm{~nm}$ from the anode region of high-current $(I=40 \mathrm{kA})$ pulsed discharges in tin vapor. It is observed that the intense multi-spike radiation in this range arises at an inductive stage of the discharge. It has been shown that the radiation spikes correlate with the sharp increase of active resistance and of pumped power, due to plasma heating by an electron beam, formed in the double layer of charged particles. It has been observed that for large number of spikes the conversion efficiency of pumped energy into radiation at double layer formation is essentially higher in comparison with collisional heating.
\end{abstract}

KEYwORDS: EUV Sources, plasma discharge, Z-pinch, EUV radiation, conversion efficiency.

\section{INTRODUCTION}

This study is devoted to the investigation of phenomena, occurring in a high-current pulse plasma diode, where the plasma with tin multi-charged ions generates extreme ultraviolet (EUV). One of the methods for the creation of high-power EUV sources for nanolithography is an application of high-current discharges in tin vapor [3, 11]. An emission source based on the dense plasma of multi-charged tin ions is advantageous against gas-filled systems because of the expected higher conversion efficiency [7] and is capable of operating at ultralow pressures. The latter is important for probability reduction of parasitic breakdowns and for reduction of losses of radiation in optical paths. Since such a source is required to have a rather high output power 9 , increasing the conversion efficiency of the supplied electric power into radiation is an important goal. Large conversion efficiency of the source is predicted for the tin [9]. For production of highcontrast nonlinear photoresists, operating in EUV range, for nanolithography above-threshold pulse intensities are required [9]. It can be provided by the narrow peak pulses (spikes) of radiation.

This work is aimed to investigation of the processes affecting the efficiency of radiation generation in highcurrent tin-vapor discharges. The results are presented on studies of the generation of intense radiation in the range of $12.2 \div 15.8 \mathrm{~nm}$ wavelength from an extended plasma diode, operating in the regime of self-sustained plasma-beam discharge [1]. The dense high-temperature plasma of multi-charged tin ions is produced by pulsed evaporation of the anode material, fast ionization of the vapor, and heating of the resulting plasma by the current and by the electron beam, formed by the double electric layer in the anode region of the diode.

\section{EXPERIMENTS}

The experimental setup dedicated to studies of the EUV yield from the plasma of multi-charged tin ions is presented in Fig. 11 The setup consists of the pulsed high-current plasma diode with the igniting electrode, the photo-detector for measurement of the integral radiation intensity, and the semicoductor detector AXUV-20 for the radiation intensity measurement of selected wavelength range. AXUV-20 detector (fabricated by International Radiation Detectors Incorporation, California) with input $\mathrm{Mo}-\mathrm{Si}$ optical filter on $12.2 \div 15.8 \mathrm{~nm}$ wavelength range was calibrated by intensity with help of the synchrotron radiation. The damped alternating current in the diode is excited between the cylindrical electrodes due to the discharge of the low-inductance capacitor bank $C_{0}$ of capacity $2.0 \mu \mathrm{F}$ at starting pressure $2 \times 10^{-6}$ Torr. The features of the applied scheme of discharge gap are the use of electrodes with a working surface of various sizes and initially the applying a positive voltage to the electrode with a small effective surface (the anode), the negative voltage - to the electrode with a larger effective surface (the cathode). At the discharge current $10 \div 40 \mathrm{kA}$ and the effective anode surface $2 \div 20 \mathrm{~mm}^{2}$ the current density reaches value about of $0.2 \div 2.0 \mathrm{MA} \mathrm{cm}^{-2}$. This leads to dense plasma formation near anode and to the necessary position stabilization of this plasma. In addition the small anode effective area provides suitable conditions of the electrical double layer formation exactly in near anode region. The double layer formation has been 


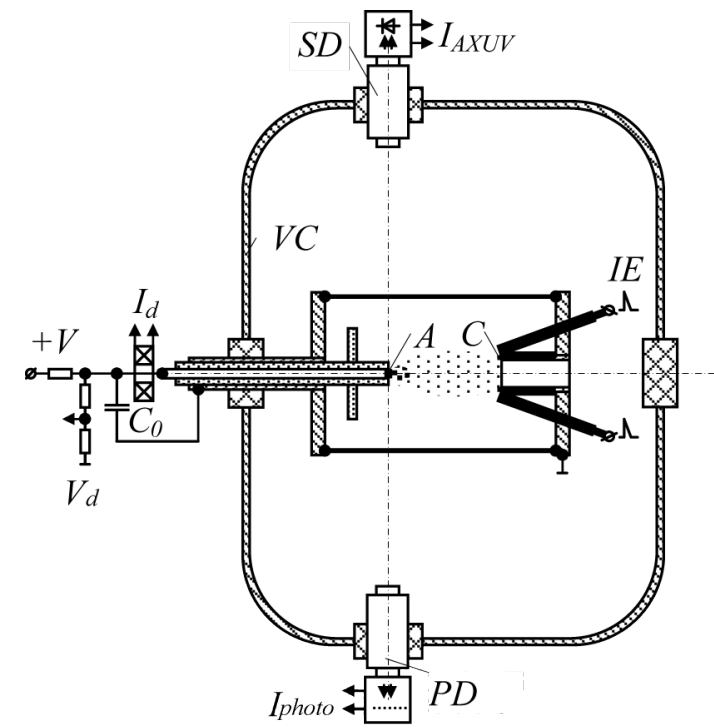

Figure 1. Scheme of the experimental setup: VC vacuum chamber, SD - detector AXUV-20, PD - photoelectron detector, A - anode, C - cathode, $I_{\mathrm{d}}-$ Rogowski coil, $V_{\mathrm{d}}$ - voltage divider, $V_{\mathrm{ig}}-$ igniting electrode.

observed in [10, 12]. In these articles at an achievement by the discharge current of some critical value all voltage is concentrated in a narrow region and a high-current electron beam is formed, which carries all discharge current. This narrow region divides the discharge on two parts. Further study of conditions of facilitation of double layer and high-current electron beam formation in such type of discharge studied by the authors of the articles [5, 4, 6. In our article the double layer formation and its dynamics are not investigated in detail. Based on the knowledge of methods for forming the double layer and its stabilization, we have created such experiment conditions to create the double layer near one electrode with a small effective surface. The using of double layer is an effective method for local plasma heating. Thus the presence in the same location of the dense plasma and its source of heating makes it possible to form dense plasma with multi-charged ions in near anode region.

The length of the discharge space can be varied from 3 to $10 \mathrm{~cm}$, the diameter of the cathode is $10 \mathrm{~mm}$ and the anode diameter equals $1.5 \mathrm{~mm}$ or $2.5 \mathrm{~mm}$, or $5 \mathrm{~mm}$. The working surface of the electrodes are covered with $0.5 \mathrm{~mm}$ thick tin layer. The side surface of a rod anode is enveloped by tubular ceramic insulator to increase the current density to the anode. The discharge voltage is from 4 to $15 \mathrm{kV}$, the current amplitude from 10 to $40 \mathrm{kA}$, the current density on the anode reaches to $0.2 \div 2.0 \mathrm{MA} \mathrm{cm}^{-2}$, the halfcycle of current oscillations is $1.7 \mu \mathrm{s}$. The discharge current and voltage are measured with Rogowski coil $I_{\mathrm{d}}$ and balanced voltage divider $V_{\mathrm{d}}$, respectively. The current through the diode is excited after filling of the discharge interval by preliminary plasma due to surface discharge on the cathode, using a igniting electrode. The pulsed voltage $0.2 \div 5.0 \mathrm{kV}$ supplies on a igniting electrode from the capacity condenser $0.025 \mu \mathrm{F}$ through thyratron and inductance of value $400 \mu \mathrm{H}$.

The integral radiation by plasma is monitored by the current measurement of photoelectrons. The photoelectrons are collected by fine-mesh grid placed at a distance of $0.5 \mathrm{~mm}$ from the photocathode. The grid is grounded, and the photocathode is at a negative potential of $-50 \mathrm{~V}$, supplied from an autonomous voltage source.

At the radiarion intensity measuring of the selected wavelength range it is used the special measures. To protect the AXUV-20 detector from the plasma and charged particle beams the inlet diafragmatic channel is located in transverse magnetic field (strength $0.2 \mathrm{~T}$, length $25 \mathrm{~cm}$ ). To exclude the effect of photoelectron current on the detector signal, we use a set of shading diaphragms and the detector is biased by $+20 \mathrm{~V}$ with respect to the channel.

The diode discharge develops in two stages. The first stage begins with a surface breakdown at the cathode and finished when the primary plasma reaches the anode. In the first stage, which lasts for $2 \div 6 \mu$ s (for the electrode gap length of $5 \mathrm{~cm}$ ), the discharge is operated in the regime of a vacuum diode with a plasma emitter. In this case, the discharge current is carried by the electron beam. Thus the working surface of the anode is preheated by the beam and the initial vapor envelope is formed. In the second stage, when the dense plasma occupied the discharge gap, the discharge switches to the plasma diode regime, in which the discharge current is determined by the parameters of the plasma and discharge circuit.

Probe measurements have shown that beetwen stages of the vacuum diode and the plasma diode the transition regime exists in which the double electric layer near the anode surface is formated. The intense electron beam are accelerated into the layer and affects on the anode surface. A high-current double electric layer exists for $0.5 \mu \mathrm{s}$. Under these conditions, the material is intensely evaporated from the anode surface, the vapor is quickly ionized and the plasma is rapidly heated due to the beam-plasma interaction. The total energy pumped in the anode plasma and the anode itself during the first half-period of the discharge current reaches $80 \%$ of the energy stored in the capacitor bank. The formation of the double layer in the transition regime is determined by inability of the discharge gap plasma to provide the high discharge current density [1, 5]. As soon as the dense plasma increases, the current is no longer limited and the double layer is disappeared. Then, the discharge operates in the inductive phase. 


\section{Results And Discussion}

From the results of the experiments it follows that the intensive radiation in the range of wavelengths $12.2 \div 15.8 \mathrm{~nm}$ arises both at the transition regime and at the inductive stage of the discharge. The peculiarity of this radiation is that there are the powerful (up to $1 \mathrm{MW}$ ) short $(100 \div 200 \mathrm{~ns})$ spikes against of the background of wide radiation pulses with duration about half-period of discharge current oscillations. The transversal dimension of the region of generation is comparable with the diameter of the anode, and its length changes depending on a discharge voltage and equals $4 \div 7 \mathrm{~mm}$. Typical waveforms of discharge current and voltage, the radiation intensity in the selected wavelength range and integral radiation versus time are shown in Fig. 2 It is seen that in the selected wavelength range there are several radiation spikes, correlating with the corresponding half-periods of the discharge current. In the first half-period there are as the wide pulse with the relatively small amplitude and a powerful spike. Whereas the pulses emitted during the second and third half-periods have only the form of the powerful shorter narrow spikes.

It is clearly seen that the radiation spikes of the selected wavelength range coincide with spike pulses of integral radiation (see Fig. 2e and 2p). Note that during first half-cycle the narrow spike of duration $200 \mathrm{~ns}$ is observed only at discharge voltage more than $7 \mathrm{kV}$. Its intensity grows with increase of the discharge voltage. More than $70 \%$ of energy, radiated during first half-cycle, is concentrated in this peak pulse. The time of occurrence of the narrow peak pulse depends on an ignition voltage. (For higher voltage the peak pulse appears earlier.)

In second and third half-cycles these radiation spikes are always observed near maximum of the current. The radiation spikes are registered at the current amplitude more than $10 \mathrm{kA}$. Their intensities grow with growth of the discharge voltage. Note that in second half-cycle of the current oscillations an additional spike-satellite of duration $200 \mathrm{~ns}$ is observed at discharge voltage $5 \div 8 \mathrm{kV}$ (Fig. $2 \mathrm{k}$ ). This pulse follows after the basic spike through 200 ns. The intensity of the spike-satellite also grows with increase of the discharge voltage. At voltage more than $8 \mathrm{kV}$ the spikesatellite disappears. Similar two spikes during the one half-period have been observed in 8 .

For effective generation of the radiation in the selected wavelength range it is required an additional energy pumping. In our case, additional energy pumping is provided by the electron beam, generated by the electric double layer which is formed periodically. In Fig. 3 the dependence of power, pumped in discharge, and corresponding pulses of radiation on time are shown. One can see that the spikes of radiation coincide with spikes of power, pumped in discharge. The different time behavior of radiation in this wavelength range in the first and subsequent half-periods can be explained as follows. In the first

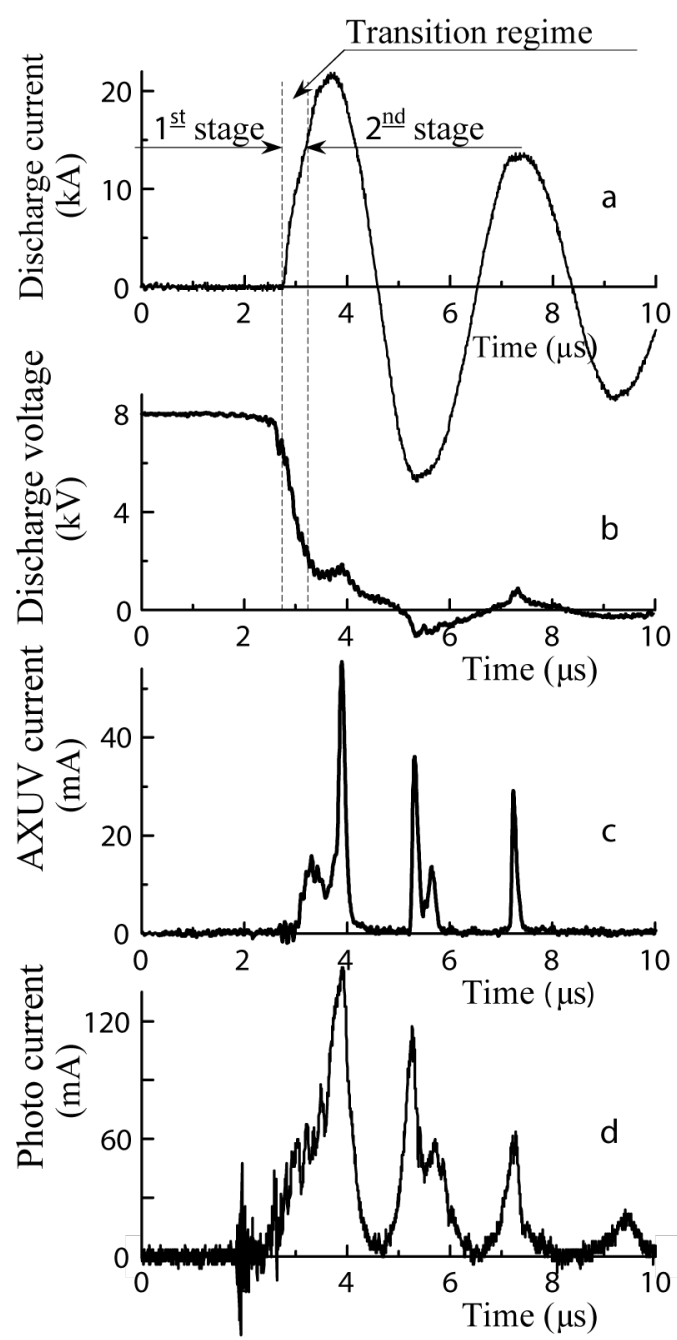

Figure 2. Waveforms of the (a) discharge current, (b) discharge voltage, (c) radiation intensity in the $12.2 \div 15.8 \mathrm{~nm}$ wavelength range, and (d) integral plasma radiation versus time, $d_{\mathrm{a}}=2.5 \mathrm{~mm}$, $V_{\mathrm{dis}}=8 \mathrm{kV}, l_{\mathrm{dis}}=5 \mathrm{~cm}$.

half-period, there is still an influx of neutral atoms into the anode region due to intense evaporation of the anode material. Moreover, since the discharge current is high, the energy distribution of plasma electrons is fairly wide. This leads to a significant collisional energy pumping; the broadening of the distribution function of ions over charge states; and, accordingly, the generation of ordinary recombination radiation within a wide spectral range with a relatively low intensity. By the end of the first half-period, the number of ions in the charge states corresponding to radiation in the $12.2 \div 15.8 \mathrm{~nm}$ wavelength range decreases.

In the second and third half-periods the plasma already exists. It is necessary only to increase the charge states (i.e., plasma temperature) of ions up to necessary ones. Moreover the plasma is relatively dense and focused during previous half-period(s). In the second and third half-periods the neutral atom flux into the anode region is much less than one in the first 

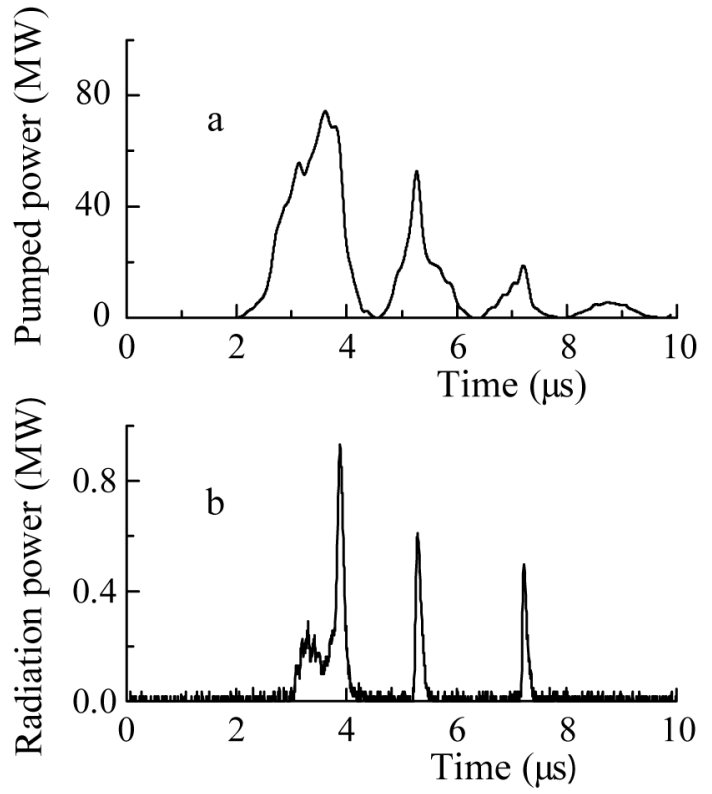

Figure 3. (a) The temporal dependences of power, pumped in discharge, (b) radiation intensity in the $12.2 \div 15.8 \mathrm{~nm}$ wavelength range; $d_{\mathrm{a}}=2.5 \mathrm{~mm}$, $V_{\mathrm{dis}}=8 \mathrm{kV}, l_{\mathrm{dis}}=5 \mathrm{~cm}$.

half-period, because existing plasma shades anode from electron beam bombardment. Thus in the subsequent half-periods the energy expenditure on formation of dense plasma with necessary charge states of ions is essentially smaller than in first half-period. After formation of plasma with necessary charge states of ions in the second and third half-periods the distribution function of ions on charge states is more narrow than in the first half-period.

We examined how the radiation intensity and the conversion efficiency of the stored energy into radiation depend on the external conditions. Fig. 4 shows how the total (within the $2 \pi$ solid angle) energy of the radiation pulses (all pulses: spike and wide) in the $12.2 \div 15.8 \mathrm{~nm}$ wavelength range depends on the energy stored in the capacitor bank. The dependence is seen to reach maximum at stored energies larger than $140 \mathrm{~J}$. It is necessary to note that this maximum in dependence of the radiation energy on the energy stored in the capacitor bank is observed at use of anode of different diameters (from 1.5 to $5 \mathrm{~mm}$ ). As it is shown in Fig. 5 for larger diameter of the anode this maximum shifts to region of larger energy stored in the capacitor bank. For smaller diameter of the anode this maximum shifts to region of smaller energy stored in the capacitor bank.

In Fig. 4 the diameter of the anode equals $2.5 \mathrm{~mm}$. Figure $4 \mathrm{p}$ also shows the energies of the radiation pulses emitted during the first, second, and third half-periods of the discharge current. At low stored energies, radiation is mainly emitted during the first half-period. As the stored energy increases, the energies of radiation pulses emitted in different halfperiods approach one another. At stored energies,
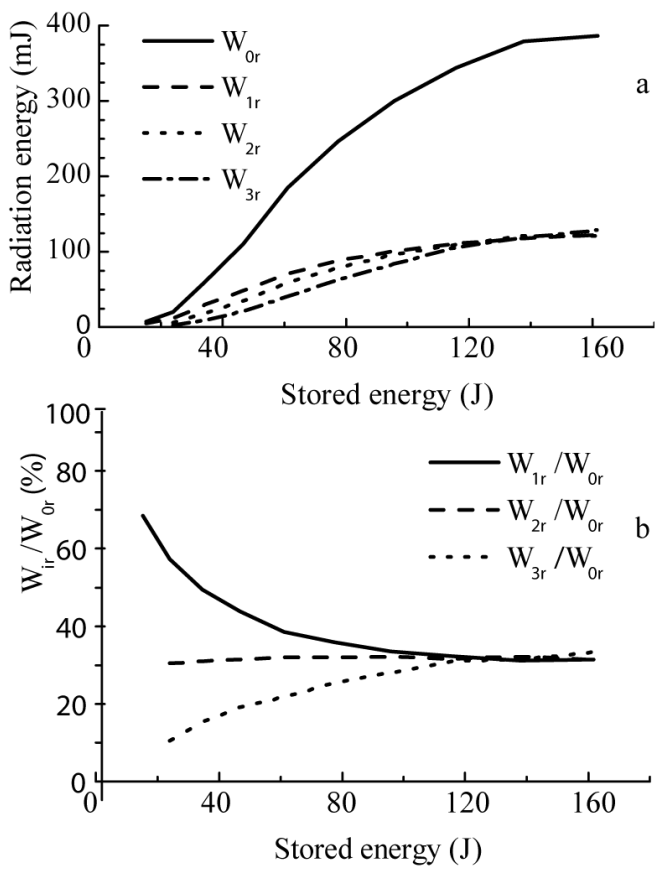

Figure 4. (a) Radiation energies (spike and wide pulse energies) in the $12.2 \div 15.8 \mathrm{~nm}$ wavelength range during first $W_{1 \mathrm{r}}$, second $W_{2 \mathrm{r}}$, third $W_{3 \mathrm{r}}$ half-periods, (b) fractions of the energy radiated in the selected wavelength range in different half-periods of the discharge current as functions of the stored energy $W_{0}$; here $W_{0 \mathrm{r}}$ is the total radiation energy, emitted in the selected wavelength range during a discharge into the $2 \pi$ solid angle; $W_{1 \mathrm{r}} / W_{0 \mathrm{r}}, W_{2 \mathrm{r}} / W_{0 \mathrm{r}}, W_{3 \mathrm{r}} / W_{0 \mathrm{r}}$ are the energy fractions emitted in the first, second, and third half-periods, respectively, $d_{\mathrm{a}}=2.5 \mathrm{~mm}, l_{\mathrm{dis}}=5 \mathrm{~cm}$.

exceeding $120 \mathrm{~J}$, they become nearly equal. Figure $4 \mathrm{~b}$ shows the relative energies of radiation pulses emitted in each half-period of the discharge current as functions of the stored energy.

Figure 6a shows the relative energy, pumped in each half-period of the discharge current, as a function of the stored energy. It is seen that the energy is mainly pumped in the first half-period, during which the anode material is intensely evaporated and neutral atoms are multiply ionized. In other words, the stored energy is mainly spent on the production of dense plasma. Very small energy in comparison with the plasma formation energy is spent on plasma support during next half-periods. Therefore, when the total stored energy is low, the rest of the energy pumped in the subsequent half-periods is low. However, the radiation intensity in those half-periods is relatively high. As the stored energy increases, the current in the second and third half-periods increases. The energy fraction pumped in the subsequent half-periods increases, thereby leading to an increase in the radiation energy.

The equalization of the absolute and relative radiation energies emitted over one half-period of the discharge current with increasing energy, as well as the fact that the radiation intensity in the second 


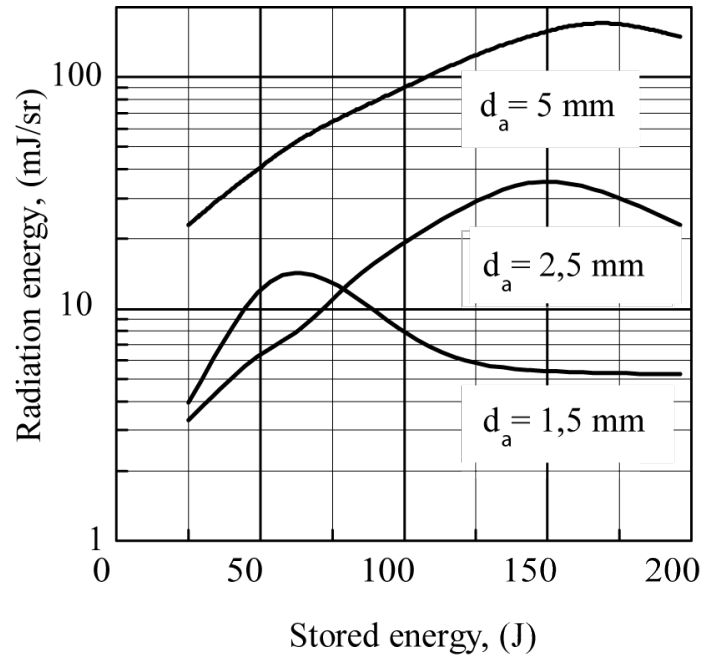

Figure 5. Radiation energies (spike and wide pulse energies) in the $12.2 \div 15.8 \mathrm{~nm}$ wavelength range as functions of the stored energy for the different anode diametres: $d_{\mathrm{a}}=1.5,2.5,5.0 \mathrm{~mm}$.

and third half-periods is high in spite of a much lower (as compared to the first half-period) energy pump, indicates that the number of particles in the dense radiating plasma remains nearly constant for a fairly long time (approximately $6 \mu \mathrm{s}$ ). This is confirmed by the results obtained in [2], where long-lived dense plasma was also observed. Thus, after the plasma has formed in the first half-period, it is supplied with energy in the subsequent half-periods, a fraction of the supplied energy being instantaneously converted into radiation.

Detailed analysis of the energy pumped in the discharge during one half-period and the energy radiated over the same time permits to find the energy conversion efficiency for each half-period. Figure $6 \mathrm{~b}$ shows the conversion efficiency of the energy pumped in the discharge during each half-period into the radiation energy in the $12.2 \div 15.8 \mathrm{~nm}$ wavelength range, and the total energy conversion efficiency $n s_{0}=W_{0 \mathrm{r}} / W_{0}$, as a function of the total stored energy. It is seen that the conversion efficiency in the second and third half-periods reaches a few percent, the energy pumped in the discharge being $10 \mathrm{~J}$. For a $2.5 \mathrm{~mm}$ diameter anode, there is an optimum at a stored energy of $80 \mathrm{~J}$ (see Fig. 6b).

A comparison of the radiation intensity in the selected wavelength range and the integral intensity shows that, when the stored energy is more above a certain value, the integral radiation intensity increases sharply, whereas the radiation intensity in the selected wavelength range of increases insignificantly. Presumably, this is related to the increasing of tin ion with the higher charge states, which leads to generation of the harder radiation and, therefore, to extra energy consumption.
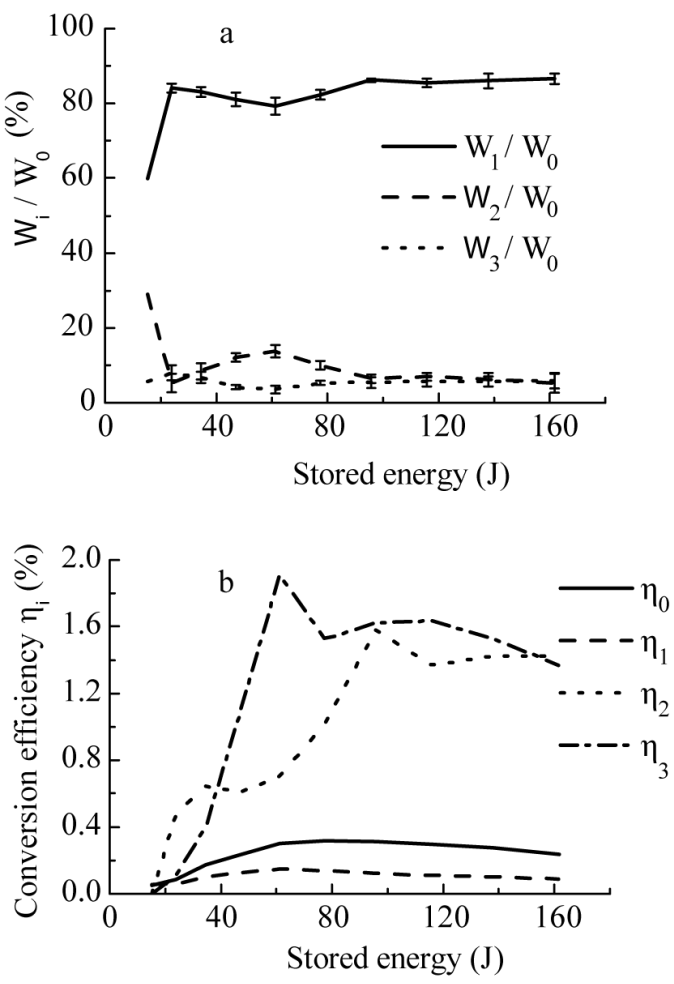

FiguRE 6. Energy fractions, pumped during one halfperiod $\left(W_{1}, W_{2}\right.$, and $W_{3}$ are the pumped energies and $W_{1} / W_{0}, W_{2} / W_{0}$, and $W_{3} / W_{0}$ are the energy fractions pumped in the first, second, and third half-periods of the discharge current, respectively); (b) conversion efficiency $\eta_{i}=W_{i \mathrm{r}} / W_{i}(i=1,2,3)$ of the energy, pumped during one half-period $W_{i}$, into the radiation energy $W_{i r}$ in the $12.2 \div 15.8 \mathrm{~nm}$ wavelength range as functions of the stored energy $W_{0} ; W_{0 \mathrm{r}}$ is the radiation energy in the selected wavelength range during all half-periods, $d_{\mathrm{a}}=2.5 \mathrm{~mm}, l_{\mathrm{dis}}=5 \mathrm{~cm}$.

\section{Conclusions}

Multi-spike radiation, important for use of highcontrast nonlinear photoresists for nanolithography, in the wavelength range $12.2 \div 15.8 \mathrm{~nm}$ from a tin vapor discharge has been observed. The duration of radiation spikes is found to be much shorter than the half-period of the discharge current.

In a narrow energy range in second half-cycle the spike-satellite is observed.

An analysis of the experimental data indicates the presence of long-lived dense plasma. When this plasma is optimally supplied with energy, radiation spikes are generated in the selected wavelength range. Analysis of the experimental data indicates that the intensity of these spikes is mainly determined by the discharge current and by the energy pumped in the discharge.

For an efficient radiation source development it is expedient that the radiating plasma be used repeatedly, because most of the stored energy is spent on plasma formation. In order to achieve quasi-steady emission, the discharge should be supplied with optimal portions of energy. 
The possibility of the power (several MW) extreme ultraviolet generation in kind of train of spikes of duration $200 \mathrm{~ns}$ in the high-current pulse plasma diode in the inductive stage of discharge development at the current density to the anode $0.2 \div 2.0 \mathrm{MA} \mathrm{cm}^{-2}$ has been shown. The power spikes of radiation are generated in conditions of plasma heating by electron beam. The electron beam is formed by double electrical layer.

The conversion efficiency of pumped energy into radiation energy at the double layer formation (beam mechanism of plasma heating) is essentially higher in comparison with ordinary heating by current, because in first case approximately all energy is pumped in very small volume.

The use of plasma diode with anode of small dimension (in comparison with cathode dimension) helps to control and stabilize in space dense plasma localization and the position of double layer formation. At this condition the space of dense plasma localization coincides with the source of its heating.

\section{REFERENCES}

[1] V. N. Borisko, et al. The formation of the low-sized high density plasma strucrures in the self-maintained plasma-beam discharge. Probl At Sci Technol ser Plasma Phys 12(6):225, 2006.

[2] V. A. Burzev, et al. About recombination nonequilibrium of plasma in low-inductive capillary discharges. JETP Letters 33(1), 2007.

[3] A. Hassanein, et al. Simulation and optimization of DPP hydrodynamics and radiation transport for EUV lithography devices. Proc of SPIE 5374:413-422, 2004.
[4] E. I. Lutsenko, N. D. Sereda, A. F. Tseluyko. Dynamic double layers in high-current plasma diodes. Journal of Technical Physics 58(7):1299-1309, 1988. (in Russian).

[5] E. I. Lutsenko, N. D. Sereda, A. F. Tseluyko, A. A. Bizukov. The dynamic characteristics of the beam-plasma self-discharge. Ukrainian Physics Journal 33(5):730-736, 1988. (in Russian).

[6] V. I. Maslov. Properties and evolution of nonstationary double layers in nonequilibrium plasma (invited lecture). In Proc. of 4th Symp. on Double Layers and Other Nonlinear Structures in Plasmas, pp. 82-92. Innsbruck, 1992.

[7] M. Masnavi, et al. Estimation of optimum density and temperature for maximum efficiency of tin ions in $\mathrm{Z}$ discharge extreme ultraviolet sources. J Appl Phys 101:033306, 2007.

[8] G. Niimi, et al. Observation of multi-pulse soft X-ray lasing in a fast capillary discharge. J Phys D: Appl Phys 34:2123, 2001.

[9] R. Seysyan. Nano-lithography SLIS by extreme ultraviolet radiation (review). Journal of Technical Physics 75(5):1-13, 2005. (in Russian).

[10] V. K. Suladze, B. A. Tshadaya, A. A. Plutto. Features of the formation of intense beams of electrons confined plasma. JETP Letters 10(6):282-285, 1969. (in Russian).

[11] Y. Tao, et al. Investigation of the interaction of a laser pulse with a preformed gaussian Sn plume for an extreme ultraviolet lithography source. J Appl Phys 101:023305, 2007.

[12] B. A. Tshadaya, A. A. Plutto, K. V. Suladze. The formation of pulse ion beams in the high-current plasma diode. Journal of Technical Physics 44(8):1779-1780, 1974. (in Russian). 